ARTICLE

Received 19 Apr 2013 | Accepted 19 Sep 2013 | Published 24 Oct 2013

DOl: $10.1038 /$ ncomms3643

OPEN

\title{
Ultrafast charge localization in a stripe-phase nickelate
}

\author{
G. Coslovich', B. Huber', W.-S. Lee ${ }^{2}$, Y.-D. Chuang ${ }^{3}$, Y. Zhu' ${ }^{1}$, T. Sasagawa ${ }^{4}$, Z. Hussain ${ }^{3}$, \\ H.A. Bechtel ${ }^{3}$, M.C. Martin ${ }^{3}$, Z.-X. Shen ${ }^{2}$, R.W. Schoenlein ${ }^{1} \&$ R.A. Kaindl ${ }^{1}$
}

Self-organized electronically ordered phases are a recurring feature in correlated materials, resulting in, for example, fluctuating charge stripes whose role in high- $T_{C}$ superconductivity is under debate. However, the relevant cause-effect relations between real-space charge correlations and low-energy excitations remain hidden in time-averaged studies. Here we reveal ultrafast charge localization and lattice vibrational coupling as dynamic precursors of stripe formation in the model compound $\mathrm{La}_{1.75} \mathrm{Sr}_{0.25} \mathrm{NiO}_{4}$, using ultrafast and equilibrium mid-infrared spectroscopy. The opening of a pseudogap at a crossover temperature $T^{\star}$ far above long-range stripe formation establishes the onset of electronic localization, which is accompanied by an enhanced Fano asymmetry of $\mathrm{Ni}-\mathrm{O}$ stretch vibrations. Ultrafast excitation triggers a sub-picosecond dynamics exposing the synchronous modulation of electronphonon coupling and charge localization. These results illuminate the role of localization in forming the pseudogap in nickelates, opening a path to understanding this mysterious phase in a broad class of complex oxides.

\footnotetext{
${ }^{1}$ Materials Sciences Division, E. O. Lawrence Berkeley National Laboratory, 1 Cyclotron Road, Berkeley, California 94720, USA. ${ }^{2}$ SIMES, SLAC National Accelerator Laboratory, Stanford University, Menlo Park, California 94025, USA. ${ }^{3}$ Advanced Light Source, E. O. Lawrence Berkeley National Laboratory, 1 Cyclotron Road, Berkeley, California 94720, USA. ${ }^{4}$ Materials and Structures Laboratory, Tokyo Institute of Technology, Kanagawa 226-8503, Japan. Correspondence and requests for materials should be addressed to G.C. (email: gcoslovich@lbl.gov) or to R.A.K. (email: RAKaindl@lbl.gov).
} 
T he self-organized segregation of charges into atomic-scale patterns is an expression of the interplay between competing interactions that governs the physics of correlated transition metal oxides ${ }^{1,2}$. In high- $T_{\mathrm{C}}$ superconductors, recent studies have observed fluctuating charge order above $T_{\mathrm{C}}{ }^{3-6}$. This raises questions about the causal relation between dynamical stripe fluctuations and the yet unexplained pseudogap phase $e^{3,5,7}$ and encourages studies of real-space charge correlations on short length and timescales. A particularly intriguing model system to explore stripe physics is the nickelate compound $\mathrm{La}_{2}{ }_{x} \mathrm{Sr}_{x} \mathrm{NiO}_{4}$ (LSNO), which is isostructural to cuprates yet exhibits real-space charge and spin stripes without superconductivity at relevant doping levels ${ }^{8,9}$. In addition, the nickel oxide class of materials has recently attracted growing attention because of novel applications in oxide electronics ${ }^{10,11}$.

The electromagnetic response of nickelates is characterized by a mid-infrared (mid-IR) gap in the optical conductivity ${ }^{12-16}$ along with strongly temperature-dependent lattice vibrations ${ }^{15,17}$. However, the relationship between stripe formation and these excitations remains unresolved, with the optical gap attributed alternatively to static charge order, fluctuating stripes or small polarons driven by strong lattice coupling ${ }^{12-19}$. In this context, ultrafast broadband spectroscopy provides tools to clarify the coupling between degrees of freedom in correlated systems by probing the time evolution of intrinsic excitations after sudden perturbation ${ }^{18-21}$. The ability of ultrafast optical pulses to suppress the long-range stripe order was, moreover, recently demonstrated in nickelates via time-resolved resonant X-ray diffraction 22,23 .

Here we combine broadband ultrafast mid-IR spectroscopy with equilibrium optical and X-ray diffraction (XRD) studies to explore the low-energy excitations of a stripe-phase nickelate and their coupling in space and time. By investigating a lightly doped LSNO sample $(x=0.25)$, we identify the mid-IR optical gap as a pseudogap feature, clearly distinct from long-range stripe ordering. We show that the pseudogap arises from electronic localization and is accompanied by a peculiar Fano asymmetry of the Ni-O stretching mode. Ultrafast photo-induced delocalization of holes triggers a strong suppression of this Fano asymmetry, whose swift recovery closely mimics the pseudogap (localization) dynamics. These results suggest the strong and rapid dependence of electron-phonon coupling on the local charge arrangement and identify electronic localization as a dynamic precursor to stripe formation.

\section{Results}

Equilibrium optical conductivity and XRD. The $\mathrm{La}_{1.75} \mathrm{Sr}_{0.25}$ $\mathrm{NiO}_{4}$ single crystal investigated by us exhibits long-range charge stripes below the charge-ordering temperature $T_{\mathrm{CO}} \approx 105 \mathrm{~K}$, accompanied by incommensurate spin order below $\approx 90 \mathrm{~K}$. Figure 1a shows the real part of the equilibrium optical conductivity $\sigma(\omega)$ at different sample temperatures, where the optical polarization is aligned along the $a b$ plane to probe the charge dynamics within the Ni-O sheets. These spectra were obtained by Fourier-transform IR spectroscopy measurements of the sample reflectivity $R(\omega)$ between $\approx 10 \mathrm{meV}-1 \mathrm{eV}$, which was converted into the complex-valued conductivity $\sigma(\omega)=\sigma_{1}(\omega)+i \sigma_{2}(\omega)$ using a Kramers-Kronig constrained variational method (see Kuzmenko ${ }^{24}$ ). With decreasing temperature, we observe a depletion of the low-frequency conductivity (Fig. 1a), as spectral weight is progressively removed and redistributed into the energy region above $0.6 \mathrm{eV}$. The transfer involves almost $80 \%$ of the spectral weight below $250 \mathrm{meV}$, as evident from the normalized conductivity in Fig. 1b. It can be quantitatively analysed by the low-energy spectral weight $W=\int_{0}^{\omega_{c}} \sigma_{1}(\omega) \mathrm{d} \omega$, where the cutoff frequency $\omega_{c} \equiv 0.6 \mathrm{eV}$ is chosen to coincide with the nearly isosbestic point in $\sigma_{1}(\omega)$.

The dramatic changes in $\sigma_{1}(\omega)$ indicate the loss of itinerancy by hole carriers concurrent with the opening of a
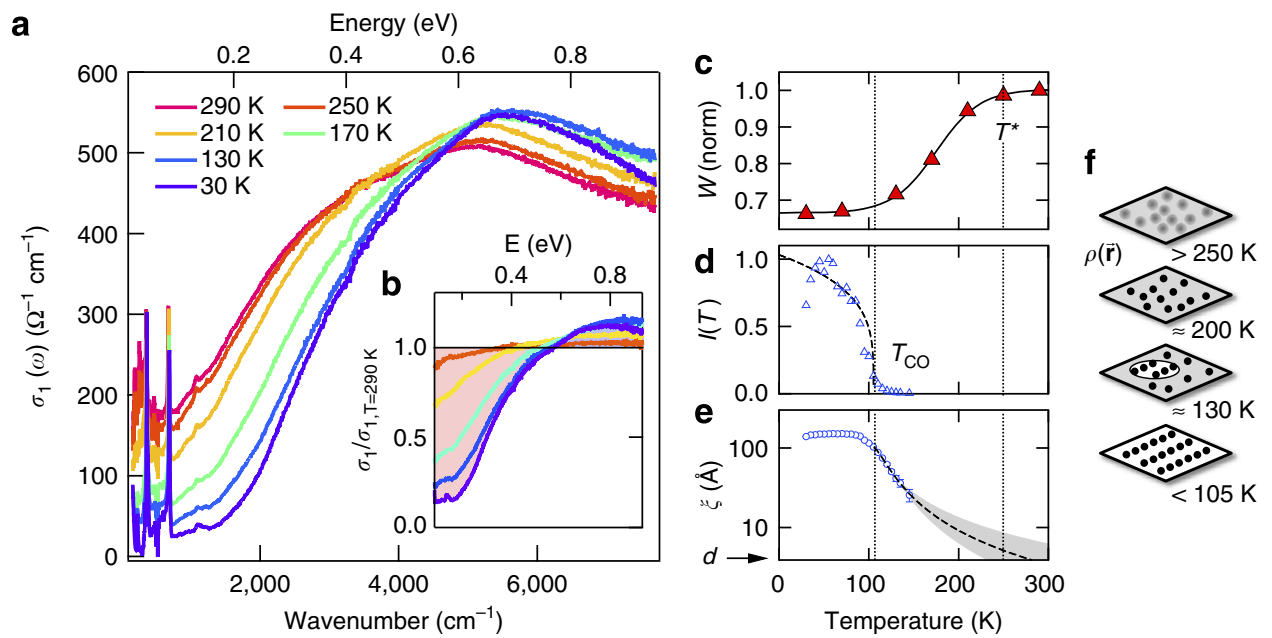

Figure 1 | Equilibrium optical conductivity and charge-order correlations. (a), In-plane optical conductivity $\sigma_{1}(\omega)$ of LSNO $(x=0.25)$ at different temperatures, and (b), data normalized to the $T=290 \mathrm{~K}$ room temperature conductivity. The shaded area indicates the difference between the normalized conductivities at room temperature and at $30 \mathrm{~K}$. (c) Temperature dependence of spectral weight below $0.6 \mathrm{eV}$, revealing a smooth crossover below $T^{\star} \approx 250 \mathrm{~K}$. Line: guide to the eyes. (d,e) Intensity and in-plane correlation length obtained from XRD from the charge-ordering superlattice at $q=(0.554,0,1)$. The correlation length is plotted on a log scale to elucidate the high-temperature trend. Dashed lines are the fits with critical scaling laws ${ }^{25}$ for the intensity $I(T) \propto\left[\left(T_{\text {co }}-T\right) / T_{\text {co }}\right]^{2 \beta}$ with $\beta=0.17 \pm 0.07$ and correlation length $\xi(T) \propto\left[\left(T-T_{\text {co }}\right) / T_{\text {co }}\right]^{-v}$ with $v=1.47 \pm 0.15$, where the statistical error represents the $95 \%$ confidence interval in extracting the fitting parameters. Within the experimental accuracy, the value of the exponent $v$ is compatible with a 2D percolative model ${ }^{25}$, where $v=1.32$. Shaded area: critical model extrapolation with twice the error bars on the fitting parameters to include possible deviations from the critical scaling law at higher temperatures. (f) Sketch of real-space charge density distribution in the Ni-O plane at different temperatures. 
low-temperature mid-IR gap. However, the spectral weight transfer $W(T)$ lacks a sharp transition at the ordering temperature $T_{\mathrm{CO}}$ (Fig. 1c). Instead, we observe a smooth crossover starting around a temperature $T^{*} \approx 250 \mathrm{~K}$ much higher than $T_{\mathrm{CO}}$. For comparison, Fig. 1d shows the intensity of X-ray diffraction resonant to the $\mathrm{Ni} L_{3}$-edge from our sample at the charge-order wave vector ${ }^{22}$. It drops quickly upon approaching $T_{\mathrm{CO}}$ and follows the known two-dimensional (2D) critical scaling (solid line, Fig. 1d), reflecting the disappearance of long-range stripes ${ }^{25}$. The high onset temperature $T^{*}$ of the spectral weight transfer in $\sigma_{1}(\omega)$ contrasts with the expectation of a charge-order gap opening at $T_{\mathrm{CO}}$ because of breaking of the translational symmetry. The features in $\sigma_{1}(\omega)$ thus instead represent a pseudogap in the midIR charge transport, governed by microscopic physics beyond the formation of long-range stripes. Our study of a lightly doped nickelate with a relatively low $T_{\mathrm{CO}}$ allows for a clear separation of these two temperature scales.

Importantly, however, in XRD a residual signal also persists beyond the transition at $T_{\mathrm{CO}}$, which indicates the presence of charge-stripe fluctuations above $T_{\mathrm{CO}}$. The correlation length $\xi$ of the fluctuations obtained from the width of the diffraction peak is shown in Fig. 1e: it rapidly shortens as the temperature increases above $T_{\mathrm{CO}}$. Extrapolating this trend above $T_{\mathrm{CO}}$ with a critical scaling model ${ }^{25}$ (dashed line in Fig. 1e) indicates that the chargeordering fluctuations reach the size of a single unit cell roughly around $T^{*}$. The survival of the optical spectral weight transfer $W(T)$ up to this temperature implies that the mid-IR pseudogap is sensitive to charge correlations at a very localized scale in the Ni-O plane which-as illustrated in Fig. $1 \mathrm{f}-$ grow into patches of short-range charge order and finally into long-range charge stripes below $T_{\mathrm{CO}}$.

Fano effect of the Ni-O stretch mode. The sharp low-energy peak in $\sigma_{1}(\omega)$ around $680 \mathrm{~cm}^{-1}$ is the Ni-O stretching mode ${ }^{14}$, which is further detailed in Fig. 2a (circles). As the crystal is cooled from room temperature to $30 \mathrm{~K}$, this phonon resonance exhibits a strong blueshift of $\approx 15 \mathrm{~cm}^{-1}$. Importantly, the lineshape becomes increasingly asymmetric at lower temperatures while it develops a broad low-energy tail. This distinct shape is characteristic of a Fano resonance, arising from quantum interference between the narrowband phonon resonance and the broadband electronic background ${ }^{26-29}$. Indeed, the phonon conductivity lineshape is well represented by the Fano model $\sigma_{\mathrm{F}}(\omega) \propto\left\{\left[(q+\varepsilon)^{2} /\left(1+\varepsilon^{2}\right)\right]-1\right\}$ indicated by the lines in Fig. 2a, in which a constant electronic background has been subtracted (more details can be found in the Supplementary Methods). Here $\varepsilon=\hbar\left(\omega-\Omega_{\mathrm{ph}}\right) / \Gamma$ is the reduced energy, where $\Gamma$ denotes the total linewidth and $\Omega_{\mathrm{ph}}$ the bare energy of the phonon. The asymmetry, and thus the interaction, is governed by the Fano parameter $q$. The temperature dependence of these parameters (Fig. 2b-d) shows that the Fano resonance develops without any sharp transition at $T_{\mathrm{CO}}$, instead evolving over a large temperature range at least comparable to $T^{*}$

Assuming a featureless electronic background, the inverse Fano parameter $1 / q$ (Fig. $2 \mathrm{c}$ ) is directly proportional to the electronphonon interaction strength $V_{\text {e-ph }}$ via the relation ${ }^{26-28}$. $q^{-1}=\pi V_{\mathrm{e}-\mathrm{ph}}\left(\sigma_{\mathrm{el}} / \sigma_{\mathrm{ph}}\right)^{1 / 2}$ where $\sigma_{\mathrm{el}}$ and $\sigma_{\mathrm{ph}}$ are the conductivities of the electronic background and phonon transition, respectively. The large change of $1 / q$ indicates a dramatic increase in electron-phonon coupling $V_{\mathrm{e}-\mathrm{ph}}$ of the Ni-O stretching mode. This effect is particularly surprising, as it occurs despite the decrease in the low-energy electronic conductivity with lower temperatures. A mere reduction in screening cannot account for this observation because the $\mathrm{Ni}-\mathrm{O}$ lineshape remains

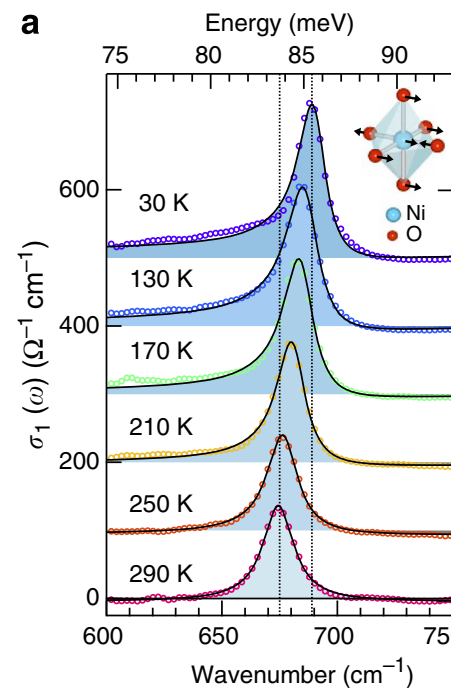

b

Figure 2 | Temperature-dependent electron-phonon coupling of the Ni-O stretch mode. (a) Optical conductivity around the Ni-O stretching mode (open circles), for different temperatures as indicated. Lines: fit with the Fano model explained in the text. Data are offset for clarity and the shaded area indicates the difference to a constant electronic background, which was removed by subtracting the conductivity at $800 \mathrm{~cm}^{-1}$.

(b-d) Temperature dependence of the parameters from the Fano model fit (symbols) showing the mode frequency $\Omega_{\mathrm{ph}}$, the Fano parameter $1 / q$ and the total linewidth $\Gamma$, respectively. Solid lines: guide to the eye. Dashed line: anharmonic line broadening remaining after considering the Fano contribution (yellow area) derived from the ultrafast modulation (see text). Error bars represent the $95 \%$ confidence interval in extracting the fitting parameters and include possible uncertainties due to the background subtraction procedure (See Supplementary Methods).

symmetric as the carrier density decreases with doping ${ }^{13}$. On the other hand, the electron-phonon interaction can sensitively depend on the spatial arrangement of holes along the interatomic bonds ${ }^{4,30}$. The picture that emerges is that the strong temperature-dependent changes in the mid-IR conductivity of LSNO track the increasing localization of charges around specific $\mathrm{Ni}$ sites upon cooling, resulting in a strongly enhanced coupling with in-plane Ni-O stretching vibrations. Such enhanced coupling can, in turn, stabilize both the on-site charge localization and stripe patterns as described theoretically ${ }^{31-33}$.

Ultrafast dynamics of pseudogap and Fano effect. To understand the interplay between these low-energy degrees of freedom in LSNO, we have investigated the ultrafast response of the mid-IR conductivity to femtosecond optical excitation. Pump pulses with 50 -fs duration and $800 \mathrm{~nm}$ wavelength were employed to perturb the charge order via $d-d$ and Ni-O charge excitations $s^{15,22,34}$, and changes of the in-plane mid-IR reflectivity were probed through $\approx 150$-fs pulses broadly tunable from $80 \mathrm{meV}$ to $1 \mathrm{eV}$. Figure $3 \mathrm{a}$ shows the induced reflectivity changes $\Delta R(\omega, \Delta t) / R(\omega)$ at $T=30 \mathrm{~K}$, as a function of probe photon energy $\hbar \omega$ and pump-probe delay time $\Delta t$. Spectral cuts at specific delay times are indicated in Fig. 3b. After excitation, the reflectivity increases for photon energies below $\approx 0.65 \mathrm{eV}$, with a concurrent decrease above this pivotal energy. The reflectivity changes occur immediately, within the time resolution, and subsequently decay back towards equilibrium on a timescale of several hundreds of femtoseconds. The time evolution of the optical conductivity can be obtained from these data, as both the equilibrium conductivity 
a

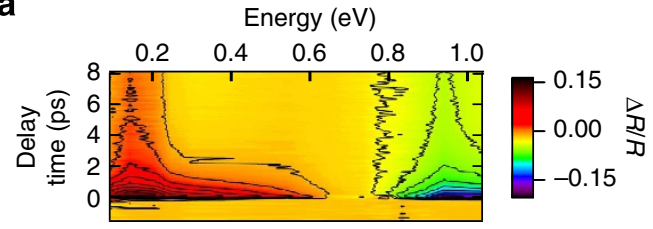

b
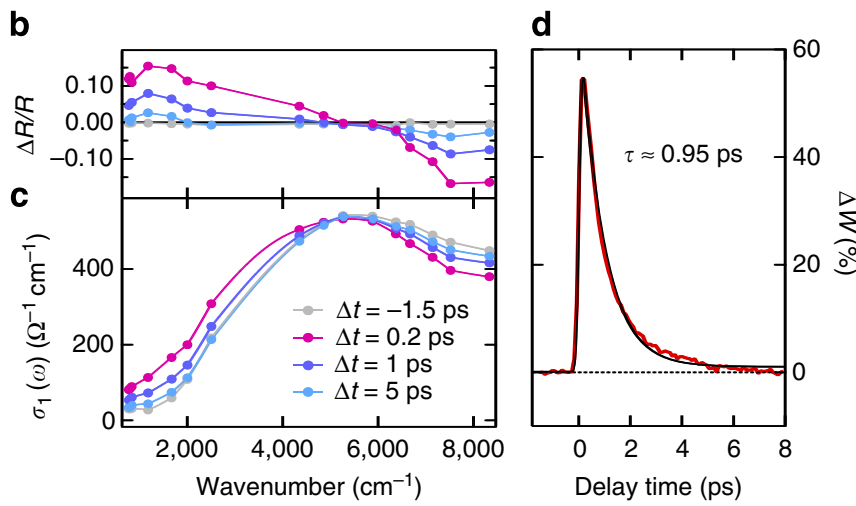

Figure 3 | Mid-infrared pseudogap dynamics. (a) Transient reflectivity variations $\Delta R / R$ as a function of energy and time delay measured at $30 \mathrm{~K}$ and for $1 \mathrm{~mJ} \mathrm{~cm}{ }^{-2}$ absorbed pump fluence. Contour lines (black) are indicated in steps of 0.02. (b) Spectral cuts of photo-induced reflectivity variations $\Delta R / R$ at representative time delays $\Delta t$, and (c) the resulting spectra of the transient conductivity $\sigma_{1}(\omega, \Delta t)$. (d) Transient change $\Delta W(t)$ of the low-energy spectral weight $W$, normalized to its full-scale variation upon heating from 30 to $290 \mathrm{~K}$. Solid line: exponential decay fit with $\tau=950$ fs convoluted with the pulse temporal profile.

and $\Delta R$ are characterized over a broad spectral range. The resulting transient conductivity spectra $\sigma_{1}(\omega, \Delta t)$ in Fig. $3 c$ reveal an increase in conductivity below $\approx 0.6 \mathrm{eV}$ and a decrease above, clearly evidencing the ultrafast filling of the mid-IR pseudogap. The corresponding spectral weight dynamics in Fig. $3 \mathrm{~d}$ shows the almost immediate (resolution-limited) recovery of $\approx 50 \%$ of the lowenergy spectral weight lost upon cooling, reflecting a fast delocalization of holes that relaxes exponentially with $\tau=950 \mathrm{fs}$ back to equilibrium.

In turn, the dynamics of lattice distortions is measured in a similar manner but now tuning the probe wavelength across the $\mathrm{Ni}-\mathrm{O}$ stretching mode resonance. The resulting conductivity spectra are shown in Fig. 4a. The peak frequency $\Omega_{\max }$ of the Ni$\mathrm{O}$ mode (squares, Fig. 4b) remains effectively unchanged after ultrafast excitation. For comparison, we plot the dynamics (solid line in Fig. 4b) expected if the phonon mode and electronic spectral weight $W(T)$ were instantaneously coupled - that is followed the dependence of Figs $1 \mathrm{c}$ and $2 \mathrm{~b}$ with a common quasiequilibrium temperature. The stark contrast underscores the nonthermal nature of the photoexcited phase and demonstrates that the $\mathrm{Ni}-\mathrm{O}$ phonon hardening in equilibrium is decoupled from pseudogap physics, arising instead from anharmonic lattice effects and screening. This absence of a line shift also rules out a significant increase in the $\mathrm{Ni}-\mathrm{O}$ phonon population or large photo-induced structural dynamics on the sub-picosecond timescale.

In contrast, the transient shape of the $\mathrm{Ni}-\mathrm{O}$ stretching mode in Fig. 4a is strongly affected by photoexcitation. A decrease in conductivity occurs in the low-energy wing concurrent with a large increase in the conductivity at the phonon peak. This corresponds to an almost complete ultrafast suppression of the Fano effect, and thus of the electron-phonon coupling. The timedependent parameters obtained from the Fano fits are plotted in Fig. $4 c, d$, showing the temporary decrease in $1 / q$ and the a

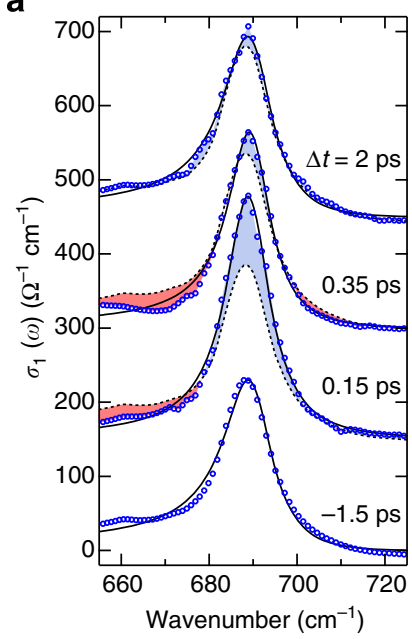

b

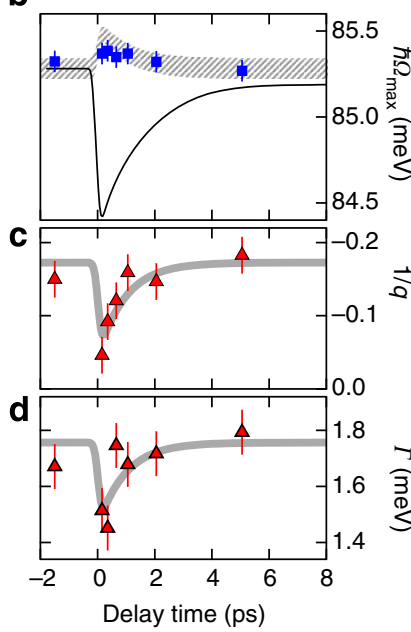

Figure 4 | Ultrafast suppression of electron-phonon coupling. (a) Nonequilibrium optical conductivity around the $\mathrm{Ni}-\mathrm{O}$ stretching mode (circles) at representative delay times after photoexcitation at $30 \mathrm{~K}$ and for $1 \mathrm{~mJ} \mathrm{~cm}{ }^{-2}$ absorbed pump fluence. Dashed lines: equilibrium conductivity, solid lines: Fano model. Blue and red shaded regions indicate a transient increase or decrease, respectively, of the conductivity. (b) Dynamics of the $\mathrm{Ni}$-O mode peak energy $\Omega_{\max }$ (squares), compared with that expected for direct coupling between the electronic spectral weight and equilibrium phonon shift as explained in the text (solid line). Hatched area: transient peak energy $\Omega_{\max }(t)=\Gamma(t) / q(t)+\Omega_{\mathrm{ph}}(T=30 \mathrm{~K})$ expected from the Fano model, taking into account the fits in panels $(\mathbf{c}, \mathbf{d})$ and their corresponding uncertainties ( $95 \%$ confidence interval). (c,d) Dynamics of the Fano coupling parameter $1 / q$ and the phonon linewidth $\Gamma$ (symbols), along with model curves (thick lines) with a single-exponential decay time fixed to $\tau=950 \mathrm{fs}$ as obtained from the spectral weight dynamics in Fig. 3d. Error bars represent the $95 \%$ confidence interval in extracting the fitting parameters and include possible uncertainties because of the background subtraction procedure (See Supplementary Methods).

linewidth. Their recovery is compatible with the spectral weight decay time as indicated by the model curves (thick lines). The resulting Fano-induced shift of the resonance peak $\Omega_{\max }(t)$ in Fig. $4 \mathrm{~b}$ (hatched line) is small and compatible with the observations. The photo-induced narrowing of the lattice resonance (Fig. 4d) further confirms the temporarily reduced electron-phonon interactions. From this, one can estimate the broadening attributable to the coupling (arrow, Fig. 2d), with the remaining linewidth modeled by multiphonon anharmonic processes (dashed line) ${ }^{29}$.

\section{Discussion}

The rapid suppression of the Ni-O Fano effect, whose recovery tracks the electronic spectral weight, suggests a strong dependence of the electron-phonon coupling on the degree of charge localization. This localized coupling can stabilize the ground state, yet it is unlikely the driving force of the relaxation dynamics, as a time-varying coupling parameter should result in a highly nonexponential, accelerating recovery of $W(t)$ that is not observed. The observed timescale can involve both electronic localization dynamics as observed in $1 \mathrm{D}$ Mott insulators ${ }^{35}$ and coupling to the lattice heat bath.

We note that the correlation between charge localization and enhanced electron-phonon coupling in our study is inferred not only from the concurrent dynamics but also from the temperature dependence of the equilibrium optical conductivity. 
This correspondence would be further strengthened by confirming the synchronous dynamics at additional excitation fluences below the present value $\left(1 \mathrm{~mJ} \mathrm{~cm}{ }^{-2}\right)$, which are inaccessible in our experiment because of signal-to-noise limitations. Future studies with high-repetition rate laser sources will allow unravelling the dependence in the low-fluence regime.

The ultrafast mid-IR response of LSNO can be compared to our recent time-resolved resonant XRD experiments, which measured the dynamics of the long-range stripe order parameter $^{22}$. The mid-IR dynamics is significantly faster, with an initial $\approx 950$-fs decay compared with the multi-picosecond recovery times of stripes at similar excitation levels. Charge localization and electron-phonon coupling thus represents the early dynamics of symmetry breaking, which precedes the slower recovery of long-range charge order at these fluences.

Our observations clarify the role of the pseudogap phase below $T^{\star}$ as a precursor that ultimately enables the organization of localized holes into stripe patterns at low temperatures. Notably, the nickelate pseudogap formation time observed here closely matches the $\approx 700$-fs pseudogap dynamics in $\mathrm{YBa}_{2} \mathrm{Cu}_{3} \mathrm{O}_{7-\delta}$ (ref. 18). Moreover, similarities with the cuprate pseudogap in momentum space were found in the highly doped metallic phase of LSNO governed by checkerboard charge fluctuations ${ }^{16}$. This opens intriguing perspectives on the pseudogap in correlated oxides and motivates further studies to explore dynamic interactions of real-space charge fluctuations and their roles in the formation of long-range order. The possibility to control charge localization and the electron-phonon coupling on ultrafast timescales can also lead to fascinating opportunities in the growing field of ultrafast oxide electronics ${ }^{36-38}$.

\section{Methods}

Samples. $\mathrm{La}_{1.75} \mathrm{Sr}_{0.25} \mathrm{NiO}_{4}$ (LSNO) single crystals were grown using the floating zone method. The crystals were first oriented using Laue diffraction and cut along the [100] surface. We use orthorhombic notation to express the crystal structure, with [100] oriented $45^{\circ}$ to the Ni-O bond direction within the $a b$ plane. The LSNO crystal surface was optically polished for the experiments. The phase-transition temperature for the long-range charge order is $\approx 105 \mathrm{~K}$ and the spin-order transition occurs at slightly lower temperature below around $\approx 90 \mathrm{~K}$.

X-ray diffraction experiments. X-Ray diffraction experiments were performed at the Advanced Light Source synchrotron, some results of which were reported in Lee $e$ e $\mathrm{al}^{22}$. The X-ray absorption spectrum (XAS) near the $\mathrm{Ni} L_{3}$-edge was first measured to calibrate the incident photon energy. Then, the resonant diffraction experiments were performed on the charge-order peak, with the X-ray photon energy tuned to the Ni $L_{3}$-edge at $851 \mathrm{eV}$.

The diffraction signal of the $\mathrm{CO}$ reflection peak $(0.554,0,1)$ at temperatures above $T_{\mathrm{CO}}$ is shown in the Supplementary Fig. $S 1$ along with corresponding fits using a squared-Lorentzian function. The peak intensity $I$ and the correlation length $\xi$ obtained from the fit coefficients (amplitude and full width at half maximum, FWHM, $w$ ) are plotted in Fig. 1 of the main text. The correlation length is calculated using the equation $\xi^{-1}=(2 \pi / a)(w / 2)$, with the lattice spacing in the $a b$ plane being $a=5.41 \AA$ and $w$ being the FWHM expressed in reciprocal lattice units (r.l.u.)

Mid-IR pump-probe experiments. Pump-probe reflectivity measurements are performed using a Ti:sapphire femtosecond laser amplifier operating at $1 \mathrm{kHz}$ repetition rate (Femtolasers Compact Pro), which generates pulses of 28 -fs duration at $\lambda=800 \mathrm{~nm}$ centre wavelength. Pump pulses are obtained by splitting off a fraction of the laser output, whereas the mid-IR and near-infrared (near-IR) probe pulses are generated via optical parametric amplification (0.5-1 eV tuning range) and cascaded difference frequency mixing of the near-IR signal and idler pulses in GaSe $(\approx 80-400 \mathrm{meV}$ range).

The pump beam is oriented at normal incidence-that is, parallel to the [100] direction-whereas the probe angle of incidence is $<10^{\circ}$. Pump and probe polarizations are oriented within the $a b$ plane of the LSNO crystal. Average pump and probe spot sizes are $d_{\text {pump }} \approx 400 \pm 50$ and $d_{\text {probe }} \approx 220 \pm 20 \mu \mathrm{m}$, respectively. Spot sizes are carefully calibrated at each wavelength through pinhole transmission measurements. The probe-to-pump intensity ratio is kept $<1: 10$.

High-precision single-shot detection is performed to sensitively measure the transient reflectivity changes as a function of the delay time between the pump and probe pulses. For this, the reflected probe energy was normalized to that of a reference pulse for each laser shot, as recorded with $\mathrm{LN}_{2}$-cooled $\mathrm{HgCdTe}$ detectors using gated integration. The time zero and pulse profiles are characterized at each wavelength through transient reflectivity pump-probe measurements of photoexcited carriers in a thin GaAs crystal. The pulse duration of the probe pulses is typically $150 \mathrm{fs}$.

The probe beam is monochromatized after interaction with the sample using a mid-IR grating spectrometer (Acton SpectraPro 2300i). This allows us to substantially improve the spectral resolution without affecting the temporal resolution of the measurement. To allow for studies of the Ni-O stretching mode, the spectral resolution in the $700 \mathrm{~cm}^{-1}$ range was set to better than $2 \mathrm{~cm}^{-1}$. In order to optimize the reproducibility of the spectral information, the data of the $\mathrm{Ni}$ $\mathrm{O}$ stretching mode dynamics are acquired with a continuous scan of the spectrum at a set of fixed delay times. For this purpose, a single mid-IR pulse is used to span the range of interest, from 650 to $800 \mathrm{~cm}^{-1}$. Standard pump-probe scans of the delay time at fixed wavelength are also used in this range to verify the accuracy of the time-domain information.

Equilibrium optical conductivity. Fourier-transform infrared (FTIR) spectroscopy measurements of the equilibrium reflectivity $R(\omega)$ were performed with a Bruker 66v/S vacuum FTIR spectrometer at Beamline 1.4.2 of the Advanced Light Source synchrotron. We employed a reflection geometry setup with $11^{\circ}$ angle of incidence and a liquid helium optical cryostat equipped with $\mathrm{KBr}$ windows. A smooth continuation outside the experimental range is obtained from known optical data of similar samples ${ }^{12,15}$

To extract the optical conductivity $\sigma(\omega)$ from the measured reflectivity data, we performed a Kramers-Kronig constrained variational fitting procedure. This procedure is inspired by the work of Kuzmenko ${ }^{25}$, and it is extended here to the case of non-equilibrium data as described further below. Following the original approach of Kuzmenko ${ }^{24}$, reflectivity data are first modeled with a standard multioscillator model of the complex dielectric function, $\varepsilon_{\bmod }(\omega)$, which reproduces the main features of the data. Second, the addition of a small variationa part, $\varepsilon_{\mathrm{var}}(\omega)$ to the total dielectric function, $\varepsilon(\omega)=\varepsilon_{\mathrm{var}}(\omega)+\varepsilon_{\bmod }(\omega)$, allows the precise fitting of the fine details of the experimental data. $\varepsilon_{\mathrm{var}}(\omega)$ is calculated as a sum of $N$ local contributions $\varepsilon_{\mathrm{var}}(\omega)=\sum_{i=1}^{N} A_{i} \varepsilon_{i}(\omega)$, where each $\varepsilon_{i}(\omega)$ contribution is centred at the corresponding anchor point $\omega_{i}$ and obeys the Kramers-Kronig transform. A function $\varepsilon_{\mathrm{i}}(\omega)$ with triangular shape in its imaginary part is the most effective in representing the local spectral weight contribution, with the real and imaginary parts ${ }^{24}$ :

$$
\begin{gathered}
\varepsilon_{1 i}(\omega)=\frac{1}{\pi}\left[\frac{g\left(\omega, \omega_{i-1}\right)}{\omega_{i}-\omega_{i-1}}-\frac{\left(\omega_{i+1}-\omega_{i-1}\right) g\left(\omega, \omega_{i}\right)}{\left(\omega_{i}-\omega_{i-1}\right)\left(\omega_{i+1}-\omega_{i}\right)}+\frac{g\left(\omega, \omega_{i+1}\right)}{\omega_{i}-\omega_{i-1}}\right], \\
\varepsilon_{2 i}(\omega)=\left\{\begin{array}{cl}
\frac{\omega-\omega_{i-1}}{\omega_{i}-\omega_{i-1}} & \omega_{i-1}<\omega<\omega_{i} \\
\frac{\omega_{i+1}-\omega}{\omega_{i+1}-\omega_{i}} & \omega_{i-1}<\omega<\omega_{i}, \\
0 & \text { otherwise }
\end{array}\right.
\end{gathered}
$$

with $g(x, y)=(x+y) \ln |x+y|+(x-y) \ln |x-y|$.

The optical conductivity $\sigma(\omega)$ and reflectivity $R(\omega)$ can be readily calculated from $\varepsilon(\omega)$. The $A_{i}$ parameters are adjusted through the fitting procedure to best reproduce the measured reflectivity, thus determining $\varepsilon(\omega)$ and $\sigma(\omega)$.

Transient optical conductivity. In the experiments, we measure the transient reflectivity change $\Delta R(\omega)$ of the photoexcited sample in a broad range of photon energies. The above variational model is expanded to calculate the corresponding conductivity change $\Delta \sigma(\omega)$ in the photoexcited sample. For this, the photoexcited dielectric function $\varepsilon_{\text {exc }}(\omega)$ is calculated as a sum of a variational dielectric function $\Delta \varepsilon(\omega)$ and the equilibrium one, $\varepsilon_{\mathrm{exc}}(\omega)=\Delta \varepsilon(\omega)+\varepsilon(\omega)$ The variational part $\Delta \varepsilon$ is then optimized through a fitting procedure to best reproduce the measured excitedstate reflectivity $R_{\text {exc }}(\omega)=\Delta R(\omega)+R(\omega)$. The effect because of a frequencydependent mismatch in penetration depths between pump and probe needs to be taken into account, as the photoexcited volume (penetration depth $\delta_{\text {pump }} \approx 150 \mathrm{~nm}$ at $\lambda=800 \mathrm{~nm})$ is generally a fraction of the probe volume $\left(\delta_{\text {probe }} \approx 550 \mathrm{~nm}\right.$ at $\lambda=5 \mu \mathrm{m})$. The penetration depth is calculated through the standard formula $\delta=\lambda / 2 \pi n_{2}$ and the full spectral dependence is shown in the Supplementary Fig. S2.

We considered an excited volume with exponentially decaying dielectric function change along the $z$ direction. This problem was solved with a transfer matrix numerical calculation of the coherent reflection and transmission of a multilayer structure with up to 1,000 layers to approximate the smooth exponentially decaying changes. The results of this calculation are closely reproduced with a less numerically intensive method, where the photoexcited volume is approximated with a single thin-film layer on top of a substrate with equilibrium optical properties. In this case, however, coherent artifacts arising from multiple reflections at the film-substrate interface must be avoided. This is achieved in the simulation by integrating over a spread of film thicknesses of $\approx 50 \%$. After confirming the consistency with the transfer matrix method, this approach was employed to extract the optical conductivity changes over a large number of frequency and time steps. 


\section{References}

1. Imada, M., Fujimori, A. \& Tokura, Y. Metal-insulator transitions. Rev. Mod. Phys. 70, 1039-1263 (1998).

2. Basov, D., Averitt, R., Van der Marel, D., Dressel, M. \& Haule, K. Electrodynamics of correlated electron materials. Rev. Mod. Phys. 83, 471-542 (2011).

3. Kivelson, S. A. et al. How to detect fluctuating stripes in the high-temperature superconductors. Rev. Mod. Phys. 75, 1201-1241 (2003).

4. Reznik, D. et al. Electron-phonon coupling reflecting dynamic charge inhomogeneity in copper oxide superconductors. Nature 440, 1170-1173 (2006).

5. Parker, C. V. et al. Fluctuating stripes at the onset of the pseudogap in the high$\mathrm{T}_{\mathrm{c}}$ superconductor $\mathrm{Bi}_{2} \mathrm{Sr}_{2} \mathrm{CaCu}_{2} \mathrm{O}_{8+\mathrm{x}}$. Nature 468, 677 (2010).

6. Torchinsky, D. H., Mahmood, F., Bollinger, A. T., Božović, I. \& Gedik, N. Fluctuating charge-density waves in a cuprate superconductor. Nat. Mater. 12, 387-391 (2013).

7. Timusk, T. \& Statt, B. The pseudogap in high-temperature superconductors: an experimental survey. Rep. Prog. Phys. 62, 61-122 (1999).

8. Tranquada, J., Kivelson, S., Uchida, S., Fink, J. \& Tajima, S. Preface. Phys. C. Superconductivity 481, 1-2 (and the following articles of the same issue) (2012).

9. Tranquada, J., Buttrey, D. \& Sachan, V. Incommensurate stripe order in $\mathrm{La}_{2-\mathrm{x}} \mathrm{Sr}_{\mathrm{x}} \mathrm{NiO}_{4}$ with $\mathrm{x}=0.225$. Phys. Rev. B 54, 12318-12323 (1996).

10. Gibert, M., Zubko, P., Scherwitzl, R., Íñiguez, J. \& Triscone, J.-M. Exchange bias in $\mathrm{LaNiO}_{3}-\mathrm{LaMnO}_{3}$ superlattices. Nat. Mater. 11, 195-198 (2012).

11. Scherwitzl, R. et al. Electric-field control of the metal-insulator transition in ultrathin $\mathrm{NdNiO}_{3}$ Films. Adv. Mater. 22, 5517-5520 (2010).

12. Ido, T., Magoshi, K., Eisaki, H. \& Uchida, S. Optical study of the $\mathrm{La}_{2-\mathrm{x}} \mathrm{Sr}_{\mathrm{x}} \mathrm{NiO}_{4}$ system: effect of hole doping on the electronic structure of the $\mathrm{NiO}_{2}$ plane. Phys. Rev. B 44, 12094-12097 (1991).

13. Bi, X.-X., Eklund, P. \& Honig, J. Doping dependence of the a-b-plane optical conductivity of single-crystal $\mathrm{La}_{2-\mathrm{x}} \mathrm{Sr}_{\mathrm{x}} \mathrm{NiO}_{4+\delta}$. Phys. Rev. B 48, 3470-3478 (1993).

14. Katsufuji, T. et al. Optical spectroscopy of the charge-ordering transition in $\mathrm{La}_{1.67} \mathrm{Sr}_{0.33} \mathrm{NiO}_{4}$. Phys. Rev. B 54, R14230-R14233 (1996).

15. Jung, J. H. et al. Optical conductivity studies of $\mathrm{La}_{3 / 2} \mathrm{Sr}_{1 / 2} \mathrm{NiO}_{4}$ : Lattice effect on charge ordering. Phys. Rev. B 64, 165106 (2001).

16. Uchida, M. et al. Pseudogap of metallic layered nickelate $\mathrm{R}_{2-\mathrm{x}} \mathrm{Sr}_{\mathrm{x}} \mathrm{NiO}_{4}$ $(\mathrm{R}=\mathrm{Nd}, \mathrm{Eu})$ crystals measured using angle-resolved photoemission spectroscopy. Phys. Rev. Lett. 106, 027001 (2011).

17. Homes, C., Tranquada, J. \& Buttrey, D. Stripe order and vibrational properties of $\mathrm{La}_{2} \mathrm{NiO}_{4+\delta}$ for $\delta=2 / 15$ : Measurements and ab initio calculations. Phys. Rev. B 75, 045128 (2007).

18. Kaindl, R. A. et al. Ultrafast mid-infrared response of $\mathrm{YBa}_{2} \mathrm{Cu}_{3} \mathrm{O}_{7-\delta}$. Science 287, 470-473 (2000).

19. Dean, N. et al. Polaronic conductivity in the photoinduced phase of $1 \mathrm{~T}-\mathrm{TaS}_{2}$. Phys. Rev. Lett. 106, 016401 (2011).

20. Dal Conte, S. et al. Disentangling the electronic and phononic glue in a high-Tc superconductor. Science 335, 1600-1603 (2012).

21. Pashkin, A. et al. Femtosecond response of quasiparticles and phonons in superconducting $\mathrm{YBa}_{2} \mathrm{Cu}_{3} \mathrm{O}_{7-\delta}$ studied by wideband terahertz spectroscopy. Phys. Rev. Lett. 105, 67001 (2010).

22. Lee, W. S. et al. Phase fluctuations and the absence of topological defects in a photo-excited charge-ordered nickelate. Nat. Commun. 3, 838 (2012).

23. Chuang, Y. D. et al. Real-time manifestation of strongly coupled spin and charge order parameters in stripe-ordered $\mathrm{La}_{1.75} \mathrm{Sr}_{0.25} \mathrm{NiO}_{4}$ nickelate crystals using time-resolved resonant X-Ray diffraction. Phys. Rev. Lett. 110, 127404 (2013).

24. Kuzmenko, A. B. Kramers-Kronig constrained variational analysis of optical spectra. Rev. Sci. Instrum. 76, 83108-83109 (2005).

25. Du, C.-H. et al. Critical fluctuations and quenched disordered two-dimensional charge stripes in $\mathrm{La}_{5 / 3} \mathrm{Sr}_{1 / 3} \mathrm{NiO}_{4}$. Phys. Rev. Lett. 84, 3911-3914 (2000).

26. Fano, U. Effects of configuration interaction on intensities and phase shifts. Phys. Rev. 124, 1866-1878 (1961).

27. Olego, D. \& Cardona, M. Self-energy effects of the optical phonons of heavily doped p-GaAs and p-Ge. Phys. Rev. B 23, 6592-6602 (1981).
28. Li, Z. et al. Structure-dependent Fano resonances in the infrared spectra of phonons in few-layer graphene. Phys. Rev. Lett. 108, 156801 (2012).

29. Mihailovic, D., McCarty, K. \& Ginley, D. Anharmonic effects and the twoparticle continuum in the Raman spectra of $\mathrm{YBa}_{2} \mathrm{Cu}_{3} \mathrm{O}_{6.9}, \mathrm{TlBa}_{2} \mathrm{CaCu}_{2} \mathrm{O}_{7}$, and $\mathrm{Tl}_{2} \mathrm{Ba}_{2} \mathrm{CaCu}_{2} \mathrm{O}_{8}$. Phys. Rev. B 47, 8910-8916 (1993).

30. Tranquada, J., Nakajima, K., Braden, M., Pintschovius, L. \& McQueeney, R Bond-stretching-phonon anomalies in stripe-ordered $\mathrm{La}_{1.69} \mathrm{Sr}_{0.31} \mathrm{NiO}_{4}$. Phys. Rev. Lett. 88, 75505 (2002).

31. Anisimov, V., Korotin, M., Zaanen, J. \& Andersen, O. Spin bags, polarons, and impurity potentials in $\mathrm{La}_{2-\mathrm{x}} \mathrm{Sr}_{\mathrm{x}} \mathrm{CuO}_{4}$ from first principles. Phys. Rev. Lett. 68, 345-348 (1992).

32. Zaanen, J. \& Littlewood, P. Freezing electronic correlations by polaronic instabilities in doped $\mathrm{La}_{2} \mathrm{NiO}_{4}$. Phys. Rev. B 50, 7222-7225 (1994).

33. Mertelj, T., Kabanov, V., Mena, J. \& Mihailovic, D. Self-organization of charged particles on a two-dimensional lattice subject to anisotropic Jahn-Teller-type interaction and three-dimensional Coulomb repulsion. Phys. Rev. B 76, 054523 (2007).

34. Simonelli, L., Huotari, S., Filippi, M., Saini, N. L. \& Monaco, G. d-d excitations and charge ordering in $\mathrm{La}_{5 / 3} \mathrm{Sr}_{1 / 3} \mathrm{NiO}_{4}$. Phys. Rev. B 81, 195124 (2010).

35. Wall, S. et al. Quantum interference between charge excitation paths in a solid-state Mott insulator. Nat. Phys. 7, 114-118 (2010).

36. Yang, Z., Ko, C. \& Ramanathan, S. Oxide Electronics Utilizing Ultrafast Metal-Insulator Transitions. Annu. Rev. Mater. Res. 41, 337-367 (2011).

37. Caviglia, A. D. et al. Ultrafast strain engineering in complex oxide heterostructures. Phys. Rev. Lett. 108, 136801 (2012).

38. Liu, M. et al. Terahertz-field-induced insulator-to-metal transition in vanadium dioxide metamaterial. Nature 487, 345-348 (2012).

\section{Acknowledgements}

We thank T. Deveraux, M. Schirò and G. Sawatzky for their interesting discussions. This research was supported by the US Department of Energy, Office of Basic Energy Sciences (DOE BES), Division of Materials Sciences and Engineering under contract DE-AC0205CH11231 at Lawrence Berkeley National Laboratory in the Ultrafast Materials Science program (G.C., B.H., Y.Z., R.W.S. and R.A.K.). The Advanced Light Source Division is supported by DOE BES under the same contract (Y.-D.C., H.B., M.C.M. and Z.H.). W.-S.L. and Z.-X.S. acknowledge the funding from the Division of Materials Sciences and Engineering, DOE BES under contract DE-AC02-76SF00515 at SLAC National Accelerator Laboratory and Stanford Institute for Materials and Energy Sciences. B.H. acknowledges fellowship support from the German Academic Exchange Service (DAAD) and participation in an exchange program with the U.C. Berkeley Nanosciences and Nanoengineering Institute.

\section{Author contributions}

G.C., B.H. and R.A.K. conceived and performed the ultrafast mid-infrared study; G.C., B.H., H.A.B. and M.C.M. implemented FTIR spectroscopy; W.-S.L, Y.-D.C., Z.H. and Z.-X.S. measured X-ray diffraction and provided the beamline; T.S. synthesized the crystals; G.C. and R.K. analysed the data and wrote the manuscript; all authors contributed to discussion and interpretation of the experimental data.

\section{Additional information}

Supplementary Information accompanies this paper at http://www.nature.com/ naturecommunication

Competing financial interests: The authors declare no competing financial interests.

Reprints and permission information is available online at http://npg.nature.com/ reprintsandpermissions/

How to cite this article: Coslovich, G. et al. Ultrafast charge localization in a stripe-phase nickelate. Nat. Commun. 4:2643 doi: 10.1038/ncomms3643 (2013).

This work is licensed under a Creative Commons AttributionNonCommercial-ShareAlike 3.0 Unported License. To view a copy of http://creativecommons.org/licenses/by-nc-sa/3.0/ 